\title{
Membrane Properties and Adrenergic Responses in Locus Coeruleus Neurons of Young Rats
}

\author{
J. T. Williams and K. C. Marshalla \\ Neuropharmacology Laboratory, Massachusetts Institute of Technology, Cambridge, Massachusetts 02139
}

Intracellular recordings were made from locus coeruleus neurons in slices taken from rats 8-26 d of age. Neurons from these animals exhibited spontaneous action potentials, which were superimposed on slow $(0.3-3 \mathrm{~Hz})$ rhythmic depolarizations. The frequency of these potentials was closely related to the age of the animals from which the slice was taken, the slowest frequencies being observed in tissues from the youngest animals. In adult animals, such rhythmic activity was only rarely observed under normal recording conditions. The rhythmic depolarizations had a slow rate of rise and fall, were 3-15 mV in amplitude, were not affected by tetrodotoxin, and were abolished in solutions that contained elevated magnesium content. When the membrane potential was hyperpolarized by passing current through the recording electrode, the depolarizing rhythmic activity persisted even at very negative potentials $(-120 \mathrm{mV})$. These depolarizations appear to be generated by the inward movement of calcium ions, probably in dendritic regions of the neuron.

Superfusion of phenylephrine caused membrane depolarizations, increased the frequency of action potentials and of the slow, rhythmic depolarizations in about $80 \%$ of the cells from young rats, whereas it had no effect or a depressant action on cells from adults. Noradrenaline hyperpolarized the cells through an alpha ${ }_{2}$-adrenoceptor and abolished the slow depolarizations. In cells from young rats, the hyperpolarization produced by noradrenaline reached a maximum and then declined, such that there was a "sag" in the membrane potential toward the resting potential following the peak of the hyperpolarization. Following the washout of noradrenaline. the membrane potential repolarized before moving toward the resting level. The decline of the membrane potential during the application of noradrenaline and the repolarization following the wash of noradrenaline were antagonized by prazosin.

The results indicate that locus coeruleus neurons exhibit alpha $_{1}$-adrenoceptor responsiveness during early stages of

\footnotetext{
Received Jan. 9, 1987; revised May 8, 1987; accepted May 8, 1987.

This work was supported by U.S. Department of Health Services Grants DA03161 and MH40416. K.C.M. was supported by a travel grant from the Medical Research Council of Canada. We with to thank Dr. R. A. North for comments on the work and manuscript.

Correspondence should be addressed to J. T. Williams at his present address: Institute for Advanced Biomedical Research, Oregon Health Sciences University, 3183 S.W. Sam Jackson Park Road, Portland, OK 97201

" Permanent address: Department of Physiology, University of Ottawa, Ottawa, Ontario, Canada.

Copyright $\odot 1987$ Society for Neuroscience $0270-6474 / 87 / 113687-08 \$ 02.00 / 0$
}

development, which is almost absent in adult animals. This might cause an enhanced responsiveness of these neurons during early postnatal periods.

The locus coeruleus (LC) is the largest of the nuclei containing noradrenergic neurons in the mammalian brain (Moore and Bloom, 1979). The responses of LC neurons to noradrenaline (NA) and adrenergic drugs have been described in animal experiments and in brain slice preparations (Aghajanian and VanderMaelen, 1982; Egan et al., 1983; Williams et al., 1985). Adrenoceptor agonists that selectively act upon beta- or alpha ${ }_{1-}$ adrenoceptors (isoprenaline and phenylephrine) have no effects, even at high concentrations, that are attributable to an action on their respective receptor subtypes. Recent studies of mouse LC neurons grown in tissue culture also indicate that these cells exhibit alpha ${ }_{2}$-adrenoceptor-mediated responses to iontophoretically applied NA. In young cultures, however, noradrenaline evokes a biphasic hyperpolarizing-depolarizing response in which the depolarization is mediated by alpha $a_{1}$-adrenoceptors, while the hyperpolarization is alpha ${ }_{2}$-adrenoceptor-mediated (Finlayson and Marshall, 1984, 1986). In view of these reports, we have studied responses of LC neurons to NA and adrenergic drugs in brain slices from young rats $(8-26 \mathrm{~d})$ to determine whether a developmentally transient alpha ${ }_{1}$-adrenoceptor responsiveness may be a general characteristic of LC cells. These studies have also permitted comparison of some of the electrophysiological properties of $L C$ neurons from young rats with already published descriptions of LC cells of adult rats (Williams et al., 1984), using the same experimental preparation. An abstract of some of the results has appeared previously (Marshall and Williams, 1986).

\section{Materials and Methods}

The methods for preparation and maintenance of the LC slice were identical to those previously described for adult rats (Williams et al., 1984). Rats of either sex in the age range 8-26 d were taken from litters with identified birth dates. These animals were anesthetized with ether and killed by a blow to the chest, or, with the younger animals, by decapitation. The cranial cavity was opened and the brain removed into cool $\left(4-10^{\circ} \mathrm{C}\right)$ physiological saline solution. A block of brain tissue (2$4 \mathrm{~mm}$ thick) containing the pons and cerebellum was prepared by cuts rostral and caudal to the pons. Serial sections were cut in an Oxford Vibratome, set for $300 \mu \mathrm{m}$, in cooled physiological saline until sections containing the LC were obtained. In younger animals, a dissection microscope was used to identify the LC and adjacent cell groups, because of the abscnce of the characteristic myelin pattern that served as a guide in older rats. A single slice containing the LC was placed in a recording chamber and superfused with physiological saline at $37^{\circ} \mathrm{C}$ and at a flow rate of $1.5 \mathrm{ml} / \mathrm{min}$. The physiological saline solution comprised (in $\mathrm{mM}$ ): $\mathrm{NaCl}, 126 ; \mathrm{KCl}, 2.5 ; \mathrm{NaH}_{2} \mathrm{PO}_{4}, 1.2 ; \mathrm{MgCl}_{2}, 1.2 ; \mathrm{CaCl}_{2}, 2.4$; glucose, $11 ; \mathrm{NaHCO}_{3}, 25$; it was gassed with $\mathrm{O}_{2} / \mathrm{CO}_{2}, 95 \% / 5 \%$.

Within the recording chamber, the LC region was localized, using a 
A

10 days

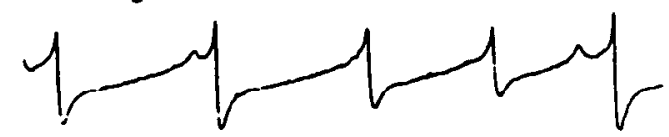

21 days

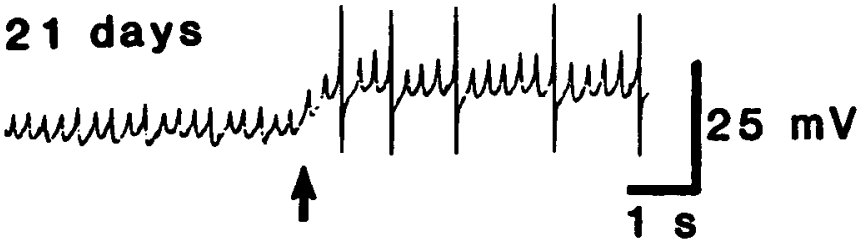

B

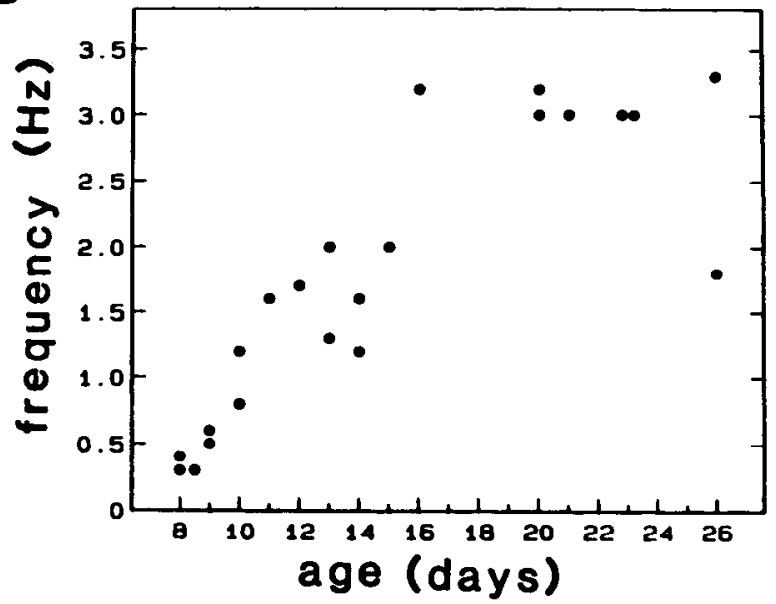

Figure 1. Age dependence of slow rhythmic calcium spikes in locus coeruleus neurons from young rats. $A$, Two examples of neurons from young rats. The slow calcium spike in the cell from a 10-d-old animal (upper trace) is slower in frequency and larger in amplitude than that in the cell recorded from a 21 -d-old animal (lower trace). The amplitude of the slow calcium spike increased slightly with depolarization from a membrane potential of about $-70 \mathrm{mV}$ toward threshold $(-55 \mathrm{mV})$. In this and other figures, the amplitudes of the full action potentials were not shown, but were distinguishable from the slow calcium spikes; full action potentials were larger in amplitude and followed by a sharp downward deflection (afterhyperpolarization). No full action potentials are present in the record from the 10-d-old animal. $B$, Summary of the frequency of the slow calcium spikes recorded from 22 cells in rats ranging in age from 8 to $26 \mathrm{~d}$. As the age of the animals from which slices were taken increases, the frequency of the slow calcium spikes increases from less than $0.5 \mathrm{~Hz}$ to over $3 \mathrm{~Hz}$.

dissection microscope, with transillumination of the slice. Recording electrodes filled with $2 \mathrm{M} \mathrm{KCl}$ and having resistances of 30-60 $\mathrm{M} \Omega$ were positioned in the $\mathrm{LC}$ using visual control. Signals were amplified using an Axoclamp II single-electrode voltage-clamp amplifier. In experiments using voltage-clamp, the potential at the headstage of the voltageclamp amplifier was monitored continuously, and a switching frequency was chosen such that the potential was flat for at least $20 \%$ of the cycle period before the potential was sampled. Membrane potential and current were recorded directly on chart paper for analysis. Drugs were applied to the slice by switching the perfusion solution to one that contained the drug in a pregassed physiological saline, by means of a 3 -way tap. There was about a $20 \mathrm{sec}$ lag between turning the 3-way tap and entry of the drug solution into the recording chamber. Drugs used were (-)noradrenaline bitartrate, cocaine $\mathrm{HCl}$, phenylephrine $\mathrm{HCl}$, TTX, muscarine (Sigma), prazosin HCl (Pfizer), idazoxan (2-(2-(1,4-benzodioxanyl))-2-imidazoline $\mathrm{HCl}$ ) (Reckitt and Colman), Try-D-Ala-GlyMePhe-Gly-ol (DAGO) (Cambridge Research Biochemicals).

\section{Results}

\section{Electrophysiological characteristics}

The membrane properties of $\mathrm{LC}$ neurons from young rats were similar in many ways to those from adult rats. That is, cells fired spontaneous action potentials at frequencies of 0.5 to $3 \mathrm{~Hz}$ in the absence of any stimulus, from a threshold of $-55 \mathrm{mV}$. Following the action potential, there was an afterhyperpolarization of $15-20 \mathrm{mV}$, with a time course of 100-300 msec, and this afterhyperpolarization merged into a slow depolarization that brought the cell to threshold again. The action potentials in younger animals were of longer duration, $1.9 \pm 0.04 \mathrm{msec}$ $(n=22)$ in $8-16 \mathrm{~d}$ animals. The amplitude of the action potentials in these animals was $71.7 \pm 1.1 \mathrm{mV}(n=22)$. With hyperpolarizing current, the frequency of spontaneous action potentials declined, as did the duration of the action potentials, and the amplitude increased. In slices from adult rats, the amplitude and duration of action potentials have been reported to be $82 \mathrm{mV}$ and $1.3 \mathrm{msec}$, respectively (Williams et al., 1984).
Steady-state current-voltage plots of LC cells, obtained under voltage-clamp from slow ramp depolarizations from $-120 \mathrm{mV}$ to $-40 \mathrm{mV}$, were similar to those in adults. The most striking difference in the properties of the cells from young animals was the presence of slow, rhythmic depolarizations, which were 3$15 \mathrm{mV}$ in amplitude and occurred at a frequency of $0.3-3.3 \mathrm{~Hz}$. These depolarizations were rarely observed in neurons recorded from adult animals, and even when they were, they were smaller in amplitude and of a higher frequency (Williams et al., 1985). In slices from 8-10 day animals, the frequency of the rhythmic depolarizations was less than $1 \mathrm{~Hz}$. As the age of the animals increased, so did the frequency of the rhythmic depolarizations, to a maximum of $3.3 \mathrm{~Hz}$ at $20-26 \mathrm{~d}$ (Fig. $1 B$ ).

Hyperpolarization of the neuron using intracellular current injection decreased the amplitude of these rhythmic depolarizations in some cells, but never abolished them (Fig. 1A). Under voltage-clamp, current oscillations having a time course identical to the slow depolarizing potentials and amplitudes of 40 $120 \mathrm{pA}$ were observed. These currents, as was true for the membrane potential oscillations, were not abolished by membrane hypcrpolarization. The slow depolarizations were not affected by TTX (600 nM) (Fig. 2). In the presence of TTX, calcium action potentials could be observed at the top of the slow depolarization. The slow depolarizations, as well as the calcium spikes, were completely abolished by superfusion with a solution to which $\mathrm{MgCl}_{2}(10 \mathrm{~mm})$ had been added $\left(\mathrm{CaCl}_{2}, 2.4 \mathrm{~mm}\right)$ (Fig. 2). This solution was found to reduce calcium currents measured under voltage-clamp (Williams et al., 1984; Williams and North, 1985 ) and to totally abolish synaptic transmission in experiments from adult animals (Egan et al., 1983). Because of their lack of voltage dependence and their sensitivity to $\mathrm{Mg}$, these siow rhythmic depolarizations were thought to result from a calcium current that was electrotonically distant in origin. These rhythmic depolarizing potentials will be referred to as slow calcium spikes. 


\section{control}

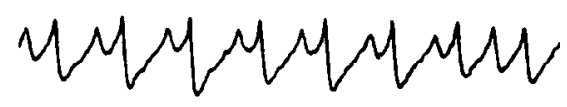

$\mathrm{Mg} 11.2 \mathrm{mM}$

\section{TTX $600 \mathrm{nM}$}

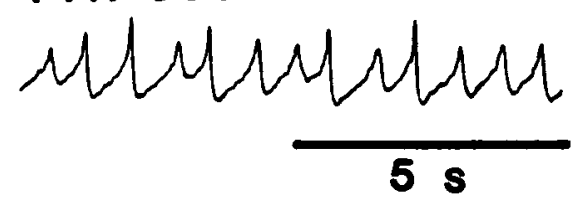

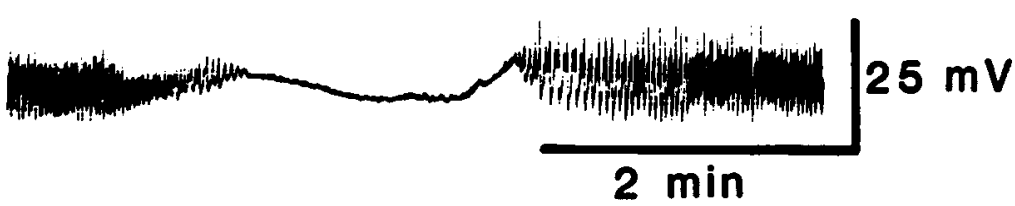

$2 \min$

Figure 2. Ionic currents underlying the rhythmic calcium spikes in young locus coeruleus neurons. Left, The slow depolarizations were unaffected by superfusion with TTX ( $600 \mathrm{nM})$. Right, The slow spikes were completely and reversibly blocked with the superfusion of a solution that contained $\mathrm{MgCl}_{2}(11.2 \mathrm{~mm})$. This cell was recorded from a slice taken from a 20-d-old rat. In this and following figures, the horizuntal bar above the recording trace indicates the period during which the superfusing solution was changed to one that contained drug.

\section{Actions of drugs}

Phenylephrine. Superfusion of phenylephrine $(30-100 \mu \mathrm{M})$ caused an increase in the firing rate or a depolarization in 18 of 23 neurons taken from young rats (Fig. 3). Phenylephrine was also found to increase the frequency of the slow calcium spikes (Fig. 7). The remaining 5 neurons were either not affected or their spontaneous activity was slightly depressed. In LC neurons taken from adult animals, phenylephrine at the same concentrations caused either no effect or a membrane hyperpolarization that was antagonized by alpha $a_{2}$-adrenoceptor antagonists (Williams et al., 1985). The action of phenylephrine in cells from young animals was not changed by idazoxan $(1 \mu \mathrm{M}$; alpha adrenoccptor antagonist), and was blocked by prazosin ( $500 \mathrm{nM}$; alpha $_{1}$-adrenoceptor antagonist) (Fig. 3). In a solution that contained an elevated $\mathrm{MgCl}_{2}$ content (11.2 $\mathrm{mm}$ ), the depolarizing action of phenylephrine was still present (Fig. 7). This solution has been previously found to block synaptic transmission in this preparation (Egan et al., 1983). These results indicated that the action of phenylephrine to increase excitability was a direct action on the neuron under study and due to an action on an

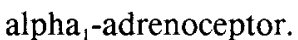

Noradrenaline. Experiments with noradrenaline also suggested that an alpha ${ }_{1}$-adrenoceptor-mediated action occurred in cells from young animals, particularly when noradrenaline was used in high concentrations. Cocaine was used in these experiments to block the reuptake of exogenously applied noradrenaline. In the absence of cocaine, the noradrenaline dose-response curve is shifted 10-30-fold to the right (Surprenant and Williams, 1987). Cocaine itself caused a decrease in the frequency of the slow calcium spikes (Fig. 4), as well as decreasing the firing rate of the neurons. Both actions were blocked by superfusion with idazoxan (1 $\mu \mathrm{M}$ ) (Fig. 6) and are thought to result from the accumulation of spontaneously released noradrenaline in the extracellular space. After superfusion with cocaine $(10 \mu \mathrm{M})$, noradrenaline (10-100 $\mu \mathrm{M})$ produced a membrane hyperpolarization that was $15-30 \mathrm{mV}$ in amplitude. The hyperpolarization reached a maximum within $20-30 \mathrm{sec}$ following the initiation of the effect (Fig. 5). The higher concentrations required less time to reach a maximum effect. These concentrations of noradrenaline (30-100 $\mu \mathrm{M}$ ) caused a hyperpolarization, the amplitude of which declined during the application (Fig. 5). In experiments on slices from 8-14-d-old animals the reduction of the noradrenaline-induced hyperpolarization was particularly marked (Figs. 5-8). In fact, immediately following the washout of noradrenaline, the membrane potential hyperpolarized before returning toward the resting level. Prior treatment of the slice with prazosin $(500 \mathrm{~nm})$ blocked the depolarizing phase of the noradrenaline effect and increased the amplitude of the hyperpolarization induced by noradrenaline (Figs. 5,8 ).

In experiments from 8-14-d-old animals, the time course of the hyperpolarization induced by noradrenaline was decreased after superfusion with prazosin, in spite of the increase in the amplitude (Figs. 5, 8). In control, following the washout of noradrenaline $(30 \mu \mathrm{M})$, complete recovery of the membrane potential required $6.0 \pm 0.84 \mathrm{~min}(3-11 \mathrm{~min}, n=12)$. This recovery time was reduced to $3.9 \pm 0.75 \mathrm{~min}(2-7 \mathrm{~min}, n=7)$ following superfusion with prazosin. In experiments on individual cells that were tested with noradrenaline before and after prazosin, the time to complete recovery was reduced to $68 \pm 4 \%$ of control $(50-81 \%, n=7)$.

\section{control}

\section{PE $100 \mu \mathrm{M}$}

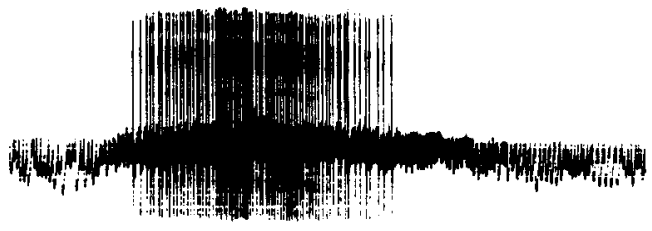

prazosin

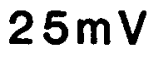

$2 \min$

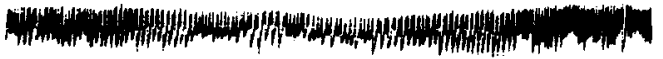

Figure 3. Phenylephrine-induced increase in firing of a locus coeruleus neuron from a 10-d-old animal. During the period indicated by the horizontal bar, phenylephrinc $(P E ; 100 \mu \mathrm{M})$ was present in the superfusion. The increase in firing rate was completely antagonized by prazosin $(500 \mathrm{nM})$. In fact, following prazosin, phenylephrine caused a decrease in the frequency of the slow calcium spikes. 


\section{control}

Figure 4. Cocaine decreased the rate of spontaneous action potentials and also the rate of the slow calcium spikes. At the arrows, continuous hyperpolarizing current $(100 \mathrm{pA})$ was applied to block the generation of full action potentials, revealing the underlying slow calcium spikes. Cocaine $(10 \mu \mathrm{M}) \mathrm{re}$ duced the frequency of action potential generation, decreased the frequency of the slow calcium spikes, but increased their amplitude. This cell was from a 21-d-old animal.

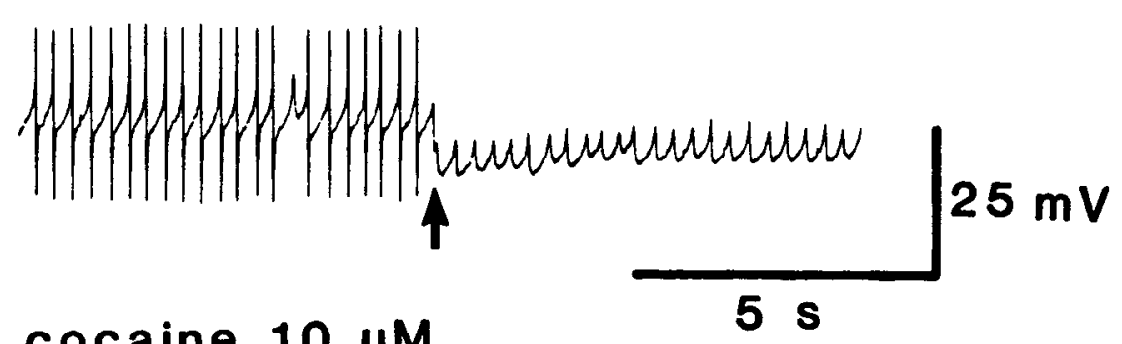

cocaine $10 \mu \mathrm{M}$

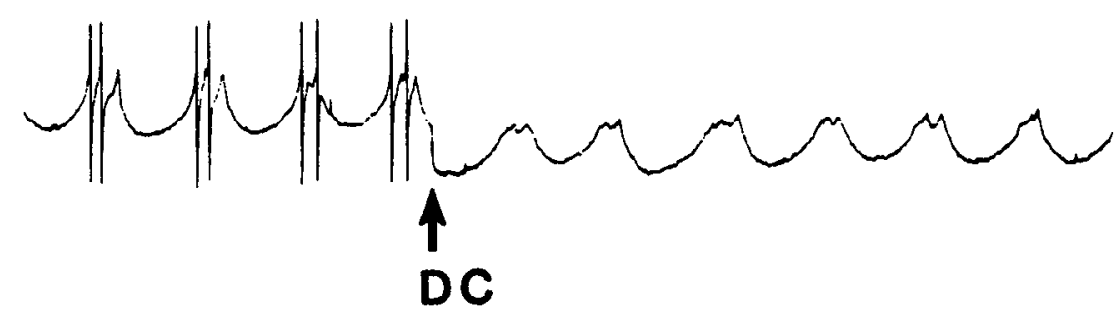

The amplitude of the "sag" in the membrane potential during the hyperpolarization induced by noradrenaline was greatest in the young animals and declined as the age of the animals increased. Experiments in cells from older animals (15-26 d) gave similar results, in that the hyperpolarization or the outward current produced by noradrenaline $(30-100 \mu \mathrm{M})$ was increased following the application of prazosin (500 nM). In fact, the outward current induced by noradrenaline $(30 \mu \mathrm{M})$ was increased following prazosin in some cells taken from adult animals (unpublished observations). These results indicate that some functional alpha ${ }_{1}$-adrenoceptors are present on LC neurons throughout development into adulthood.

Opiates. Activation of $\mu$-opioid receptors with DAGO caused a membrane hyperpolarization $20-30 \mathrm{mV}$ in amplitude. In young animals the amplitude of the DAGO- $(3 \mu \mathrm{M}$; top of the concentration curve) induced hyperpolarization was similar to that of the response to noradrenaline $(10-30 \mu \mathrm{M})$ following treatment of the slice with prazosin (500 nM; Fig. 8). Opioid and alpha ${ }_{2}^{-}$ adrenoceptor agonists have been shown to activate a single potassium conductance (Andrade and Aghajanian, 1985; North and Williams, 1985). A similar situation seems to exist in cells from young animals.

The voltage dependence of the noradrenaline- and DAGOinduced currents in cells from young animals was studied under voltage-clamp. Steady-state current-voltage plots were constructed using a slow ramp depolarizations $(-130$ to $-40 \mathrm{mV})$ in the absence and presence of both noradrenaline and DAGO (Fig. 9). In cells from young animals, the outward current induced by both agents declined as the membrane potential was held at more negative potentials, but never reversed in polarity even at potentials up to $-130 \mathrm{mV}$. This contrasts with experiments in cells from adult animals, where the potassium conductance increased by opioids and alpha $\mathrm{a}_{2}$-adrenoceptor agonists had a reversal potential near the potassium equilibrium potential (Egan et al., 1983; Williams et al., 1984; North and Williams, 1985).

\section{NA $30 \mu M$}

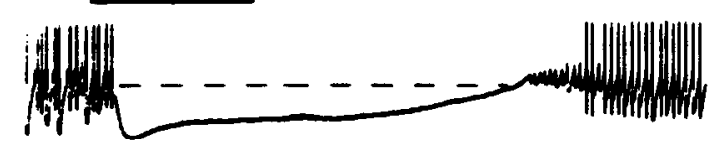

\section{NA $100 \mu M$}

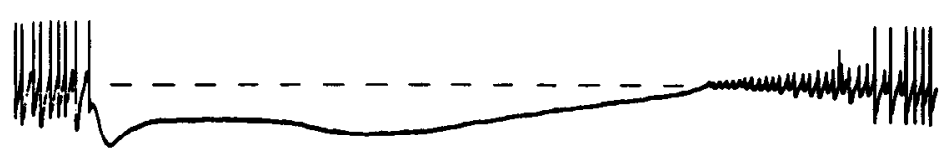

\section{prazosin}
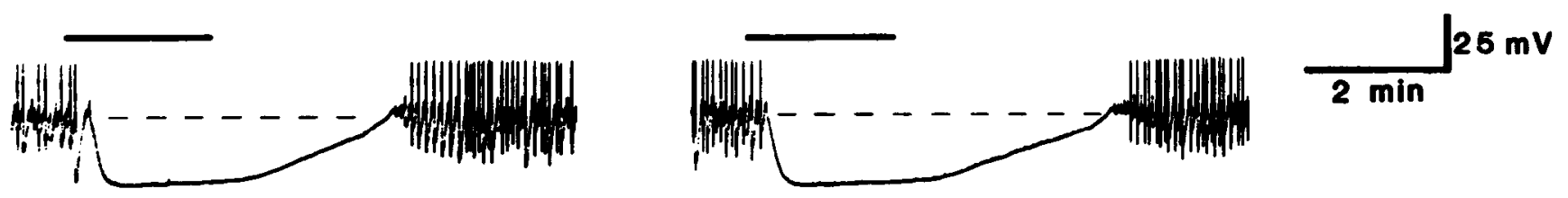

Figure 5. Noradrenaline $(N A)$ hyperpolarizations were increased in amplitude and decreased in duration following superfusion with prazosin. This experiment was carried out in the presence of cocaine $(10 \mu \mathrm{M})$. Top, Noradrenaline produced inhibition of fring, an inhibition of the slow calcium spikes, and a hyperpolarization the duration of which was dependent on the concentration applied. The hyperpolarization declined during the application of noradrenaline. Following the wash of noradrenaline, the membrane potential rehyperpolarized before returning toward the resting level. Bottom, Following superfusion with prazosin $(500 \mathrm{nM})$, noradrenaline caused a membrane hyperpolarization that was greater in amplitude and shorter in duration for both concentrations of NA. This cell was from a 9-d-old animal. 


\section{control}

\section{NA $30 \mu M$}

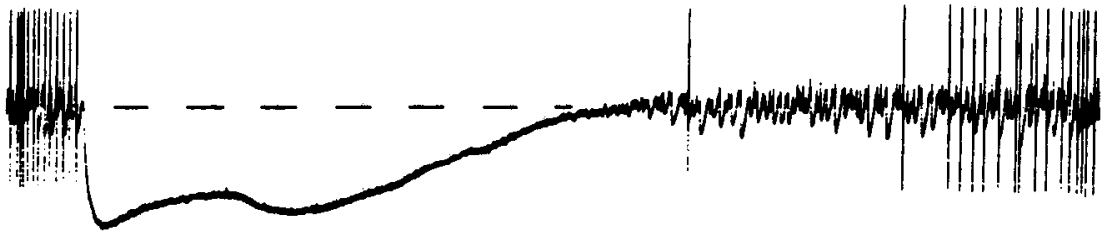

\section{idazoxan $1 \mu \mathrm{M}$}

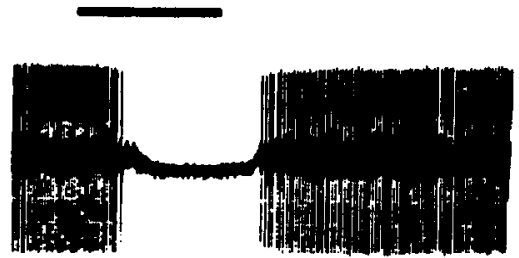

idazoxan $3 \mu \mathrm{M}$

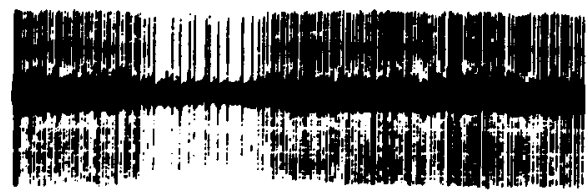

In some experiments, the action of phenylephrine was examined during the simultaneous action of DAGO (1-3 $\mu \mathrm{M})$. Under these conditions, phenylephrine depolarized the membrane $(3-5 \mathrm{mV})$ even in cells where it had had no apparent depolarizing action before application of DAGO. These observations were obtained from young rats $(15-26 \mathrm{~d}, n=4)$ as well as from adults $(n=3)$.
NA $100 \mu M$

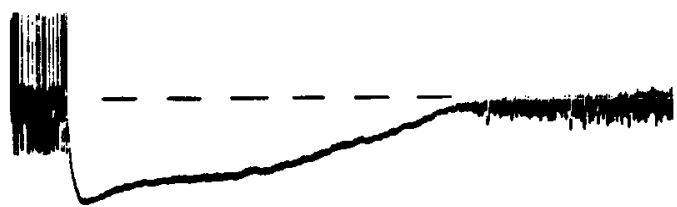

Mg $11.2 \mathrm{mM}$

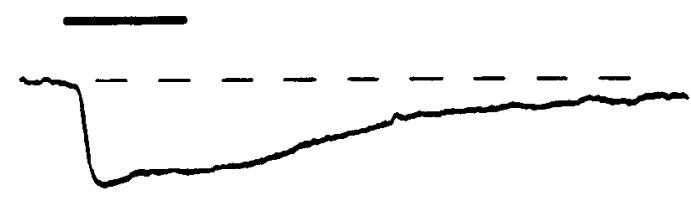

$25 \mathrm{mV}$

$2 \min$
Figure 6. The noradrenaline-induced hyperpolarization was reduced by idazoxan. Top, Noradrenaline ( $N A$ ) caused a hyperpolarization that declined during the application period. Following washout of the noradrenaline, the membrane potential repolarized before returning to the resting level. Bottom, The same concentration of noradrenalinc inhibited firing but caused only a small hyperpolarization in the presence of idazoxan (1 and $3 \mu \mathrm{M}$ ). Note that not only was the initial hyperpolarization absent, the later variations in potential were also eliminated by idazoxan. From a 13-d-old animal.

\section{Discussion}

The excitatory action of phenylephrine on neurons taken from young rats and the blockade of this action by prazosin indicate

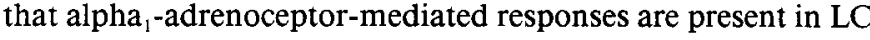
neurons from young animals. LC neurons recorded from adult animals were not affected or were hyperpolarized by phenyl-
PE $30 \mu \mathrm{M}$

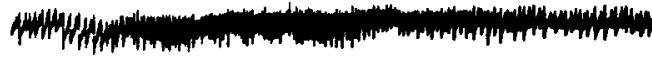

$\mathrm{Mg} 11.2 \mathrm{mM}$

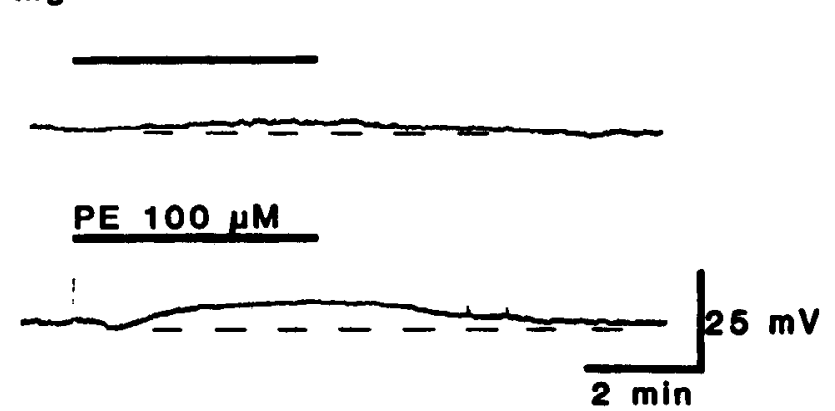

Figure 7. Noradrenaline and phenylephrine act in the presence of high-Mg solutions. Left, Noradrenaline $(N A)$ hyperpolarized the membrane and blocked the slow calcium spikes. The hyperpolarization was not changed after superfusion of the slice with a solution of elevated $\mathrm{MgCl}_{2}$. From a 15-d-old animal. Right, Phenylephrine $(P E)$ increased the frequency of the slow calcium spikes in control solutions (upper trace). After addition of $\mathrm{MgCl}_{2}$ to the superfusion solution, phenylephrine produced a 2-3 mV depolarization at $30 \mu \mathrm{M}$ (middle trace) and a 3-5 mV depolarization at $100 \mu \mathrm{M}$ (lower trace). Cocaine $(10 \mu \mathrm{M})$ was present throughout this experiment. From an 8-d-old animal. 


\section{control}

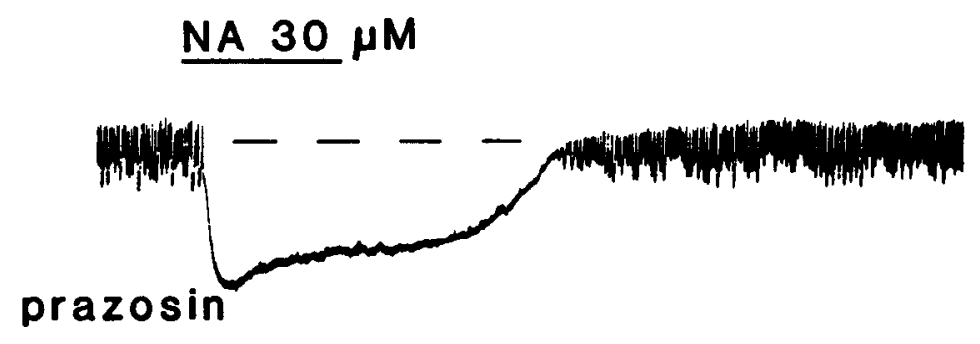

Figure 8. Adrenergic and $\mu$-opioid receptor activation caused similar peak hyperpolarizations, but the noradrenaline $(N A)$ response was reduced after reaching a maximum. This entire experiment was carried out in the presence of cocaine $(10 \mu \mathrm{M})$. Following superfusion with prazosin, the hyperpolarization induced by noradrcnaline $(30 \mu \mathrm{M}$, middle record $)$ was identical in amplitude to that induced by DAGO ( $3 \mu \mathrm{M}$, bottom record $)$. From a 21 -d-old animal.
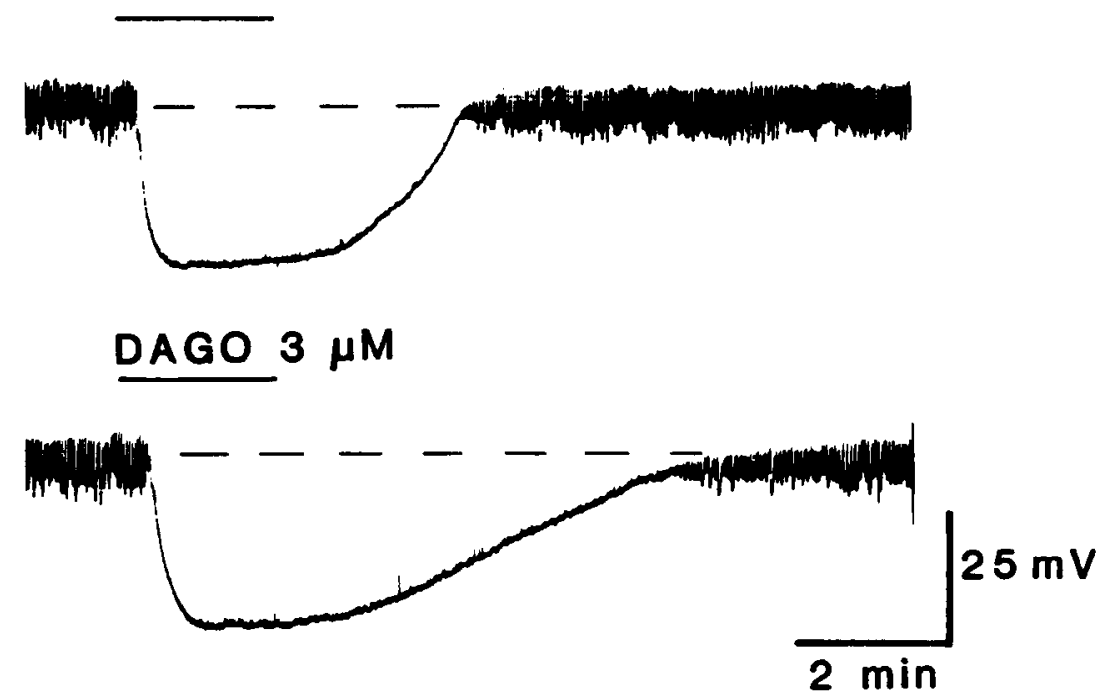

ephrine, indicating that the alpha $a_{1}$-adrenoceptor responsiveness decreased with age. This confirms findings obtained using mouse brain cultures of LC neurons, which have demonstrated developmentally transient alpha $a_{2}$-adrenergic responses (Finlayson and Marshall, 1986). This action mediated by alpha $a_{1}$-adrenoceptors results from a direct action on the cell, since it is still present following procedures that are known to block synaptic transmission. The alpha $a_{1}$-adrenoceptors arc present in the LC during a period that is also characterized by other developmentally transient membrane properties, i.e., the presence of slow calcium-dependent variations of membrane potential. This rhythmic activity results from the entry of calcium, which either is not sensitive to membrane potential, which seems unlikely, or is electrotonically distant (dendritic or from electrotonically coupled neurons) in origin. That there is a large, electrotonically distant component in the actions of $\mu$-opioid and alpha $a_{2}$-adrenoceptor agonists was suggested by the observation that reversal potentials for these agents were consistently more negative than the potassium equilibrium potential. With development, the membrane properties and the potassium conductance, increased by $\mu$-opioids and alpha $a_{2}$-adrenoceptors that are recorded in the soma, appear to have less of an electrotonically distant component. In most LC neurons from adult animals, there is no cvidence of the rhythmic depolarizations seen in young animals, and reversal potentials for noradrenaline and opioids approximate the potassium equilibrium potential (Egan et al., 1983; Williams et al., 1984; North and Williams, 1985). It should be noted that the alpha ${ }_{1}$-adrenoceptor-mediated depolarization does not totally disappear with development.
The hyperpolarizing response to noradrenaline was consistently increased in amplitude following blockade of alpha $a_{1}$-adrenoceptors with prazosin, even in adult animals (J. T. Williams, unpublished observations). The magnitude of this action varied from cell to cell and could be predicted by comparing the amplitude of the noradrenaline ( $30 \mu \mathrm{M}$ ) hyperpolarization with that of the response to $\mathrm{Met}^{\mathrm{s}}$-enkephalin $(\mathrm{ME} ; 30 \mu \mathrm{M}$ ) or DAGO (3 $\mu \mathrm{M})$. In cclls whcre the noradrenaline and opioid responses were almost equal, there was little change in the noradrenaline response following prazosin. However, when the response to noradrenaline was small compared to that of $\mathrm{ME}$, noradrenaline responses following prazosin increased, becoming about equal to those generated by the opioid. It was also observed that LC cells from adult animals were depolarized by phenylephrine following a conditioning hyperpolarization with opioids. This action occurred in cells that were not affected by phenylephrine alone. These results indicate that a small alpha $a_{1}$-adrenoceptor action is maintained with development. The site of origin of the alpha $a_{1}$-adrenoceptor action in both adults and the young rats remains to be determined.

Idazoxan (1-3 $\mu \mathrm{M})$ antagonized the hyperpolarization produced by noradrenaline in LC neurons from young rats. It is interesting to note that even in cells where a large alpha $a_{1}$-adrenoccptor-mcdiated component of the noradrenaline responses was observed, after blockade of alpha $a_{2}$-adrenoceptors with idazoxan no excitatory component was left. This is different from the results in cultured mouse LC neurons, where in younger cultures an alpha $a_{1}$-adrenoceptor-mediated depolarization was present following blockade of the alpha $\mathrm{a}_{2}$-adrenoceptor-mediated 


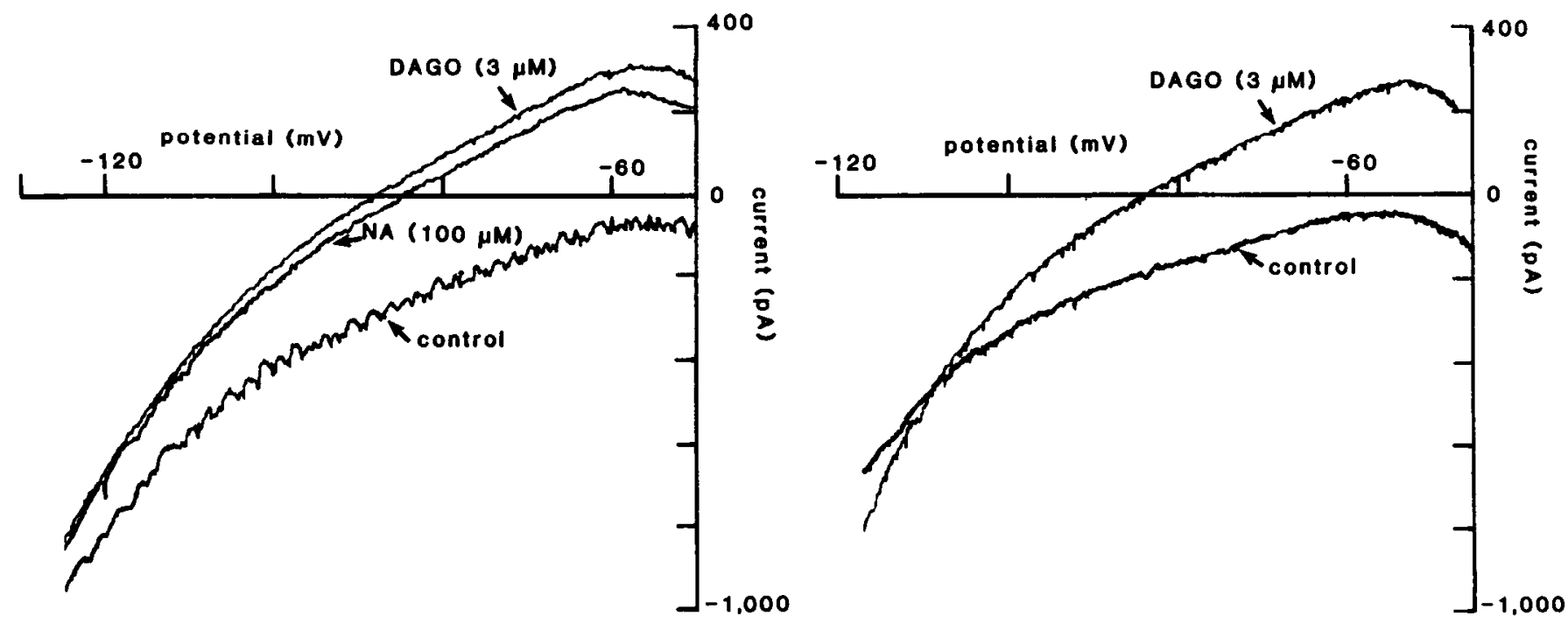

Figure 9. Steady-state current-voltage plots from a young animal (left) and an adult (right). Right, Two superimposed steady-state current-voltage plots of an LC neuron in the absence and presence of DAGO $(3 \mu \mathrm{M})$. Relative to the control, DAGO produced an outward current in the potential range from -110 to $-40 \mathrm{mV}$. The reversal potential was $-110 \mathrm{mV}$ and an inward current flowed at potentials more negative than $-110 \mathrm{mV}$. Noradrenaline typically produced the same action (not shown). Left, Three superimposed steady-state current-voltage plots: a control; in the presence of noradrenaline $(N A ; 100 \mu \mathrm{M})$; and in the presence of DAGO $(3 \mu \mathrm{M})$. Relative to the control, noradrenaline and DAGO produced outward currents that were similar throughout the potential range. Neither the DAGO nor the noradrenaline currents reversed with membrane hyperpolarization to levels more negative than $-120 \mathrm{mV}$.

hyperpolarization (Finlayson and Marshall, 1986).

Studies of adrenergic receptor binding suggest that a transient presence of alpha ${ }_{1}$-adrenoceptors may underlie the enhanced alpha ${ }_{1}$-responsiveness of LC neurons in young animals. An autoradiographic study by Young and Kuhar (1980) indicated that in the LC of adult rats, alpha $a_{2}$-adrenoceptors were present in high concentration, while alpha ${ }_{1}$-adrenoceptors were not. Using a different alpha ${ }_{1}$-adrenoceptor-binding ligand (125I-HEAT), however, Jones et al. (1985a) have reported quite dense binding in LC of mature rats. Other studies have suggested that, while in some brain locations, alpha ${ }_{1}$-adrenoceptor binding increases with early postnatal development, in others binding is high in the first 2 weeks of postnatal life and decreases to low levels thereafter (Johnson et al, 1984; Jones et al., 1985b). The latter pattern would correspond to our observations of phenylephrine responsiveness in LC. Curiously, the pattern of increasing alpha ${ }_{1}-$ adrenoceptor binding with development has been assigned to the LC (Jones et al., 1985b). In view of the discrepancy in results

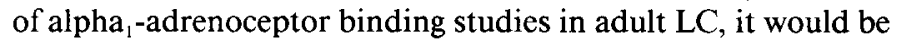
interesting to test developmental changes in binding of a ligand such as prazosin, which was shown in our studies to block the

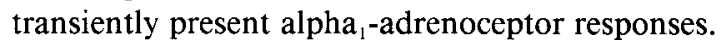

It is possible that developmentally transient alpha ${ }_{1}$-adrenoceptor responsiveness could explain developmental changes in behavioral responses to adrenergic agents. Injections of clonidine, which can evoke alpha ${ }_{1}$ - as well as alpha ${ }_{2}$-adrenoceptor responses, produce locomotor hypoactivity in adult rats, but hyperactivity in 1-2-week-old rats (Reinstein and Isaacson, 1977). The hypoactive response of adults is antagonized by adrenergic blockers that have some selectivity for alpha $a_{2}$-adrenoceptors (yohimbine and piperoxan) (Nomura et al., 1980). The hyperactive responses of young rats have also been tested using adrenergic antagonists, and while the results favored the likelihood of mediation by alpha ${ }_{2}$-adrenoceptors, the possibility

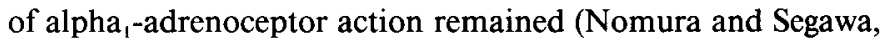
1979).

That there is a physiological action of noradrenaline in the locus coeruleus in vivo in adult animals has been demonstrated on the late component of the inhibitory period following antidromic and orthodromic stimulation of LC cells (Ennis and Aston-Jones, 1986). The possible physiological significance of the observed developmental differences in noradrenaline responsiveness is unclear. The effects of cocaine indicate that noradrenaline released from the LC neurons can affect activity of

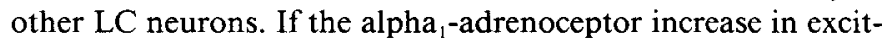
ability is more pronounced in early postnatal stages, an increased activity and/or responsiveness of LC neurons would be expected. It is interesting, therefore, that the results of a recent study of 1-3 d-old-rats suggest that LC neurons at this stage are highly sensitive to sensory activation (Kimura and Nakamura, 1985).

\section{References}

Aghajanian, G. K., and C. P. VanderMaelen (1982) Alpha -adreno- $^{-}$ ceptor-mediated hyperpolarization of locus coeruleus ncurons: Intracellular studies in vivo. Science 215: 1394-1396.

Andrade, R., and G. K. Aghajanian (1985) Opiate- and alpha $a_{2}$-adrenoceptor-induced hyperpolarizations of locus coeruleus neurons in brain slices: Reversal by cyclic adenosine $3^{\prime}: 5^{\prime}$-monophosphate analogues. J. Neurosci. 5: 2359-2364.

Egan, T. M., G. Henderson, R. A. North, and J. T. Williams (1983) Noradrenaline-mediated synaptic inhibition in rat locus coeruleus neurones. J. Physiol. (Lond.) 345: 477-488.

Ennis, M., and G. Aston-Jones (1986) Evidence for self- and neighbormediated postactivation inhibition of locus coeruleus neurons. Brain Res. 374: 299-305.

Finlayson, P. G., and K. C. Marshall (1984) Hyperpolarization and age-dependent depolarization responses of cultured locus coeruleus neurons to noradrenaline. Dev. Brain Res. 15: 167-175.

Finlayson, P. G., and K. C. Marshall (1986) Locus coeruleus neurons in culture have a developmentally transient alpha ${ }_{1}$-adrenergic response. Dev. Brain Res. 25: 292-295. 
Johnson, A. E., B. Nock, H. Ryder, and H. Feder (1984) Hypothalamic and preoptic area alpha ${ }_{1}$-receptor concentrations increase during postnatal maturation of male guinea-pigs. Neuroendocrinology 38: 243247.

Jones, L. S., L. L. Gauger, and J. N. Davis (1985a) Anatomy of brain

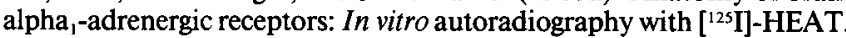
J. Comp. Neurol. 231: 190-209.

Jones, L. S., L. L. Gauger, J. N. Davis, T. A. Slotkin, and J. V. Bartolome (1985b) Postnatal development of brain alpha ${ }_{1}$-adrenergic receptors: In vitro autoradiography with [ $\left.{ }^{125} \mathrm{I}\right]-\mathrm{HEAT}$ in normal rats and rats treated with alpha-difluoromethylornithine, a specific, irreversible inhibitor of ornithine decarboxylase. Neuroscience 15: 1195-1202.

Kimura, F., and S. Nakamura (1985) Locus coeruleus neurons in the neonatal rat: Electrical activity and responses to sensory stimulation. Dev. Brain Res. 23: 301-305.

Marshall, K. C., and J. T. Williams (1986) Rhythmic activity and adrenergic responses in locus coeruleus neurons from young rats. Soc. Neurosci. Abstr. 16: 377.

Moore, R. Y., and F. E. Bloom (1979) Central catecholamine neuron systems: Anatomy and physiology of the norepinephrine and epinephrine systems. Annu. Rev. Neurosci. 2: 113-168.

Nomura, Y., and T. Segawa (1979) The effect of alpha-adrenoceptor antagonists and metiamide on clonidine-induced locomotor stimulation in the infant rat. Br. J. Pharmacol. 66: 531-535.
Nomura, Y., K. Oki, and T. Segawa (1980) Pharmacological characterization of central alpha-adrenoceptors which mediate clonidineinducing locomotor hypoactivity in the developing rat. Naunyn Schmiedeberg's Arch. Pharmacol. 311: 41-44.

North, R. A., and J. T. Williams (1985) On the potassium conductance increased by opioid in rat locus coeruleus neurones. J. Physiol. (Lond.) 364: $265-280$.

Reinstein, D. K., and R. L. Isaacson (1977) Clonidine sensitivity in the developing rat. Brain Res. 135: 378-382.

Surprenant, A., and J. T. Williams (1987) Inhibitory synaptic potentials recorded from mammalian neurones prolonged by blockade of noradrenaline uptake. J. Physiol. (Lond.) 382: 87-103.

Williams, J. T., and R. A. North (1985) Catecholamine inhibition of calcium action potentials in rat locus coeruleus neurones. Neuroscience 14: 103-109.

Williams, J. T., R. A. North, S. S. Shefner, S. Nishi, and T. M. Egan (1984) Membrane properties of rat locus coeruleus neurones. Neuroscience 13: 137-156.

Williams, J. T., G. Henderson, and R. A. North (1985) Characterization of alpha ${ }_{2}$-adrenoceptors which increase potassium conductance in the rat locus coeruleus neurones. Neuroscience 14:95-101.

Young, W. S., and M. J. Kuhar (1980) Noradrenergic alpha ${ }_{1}$ - and alpha $_{2}$-receptors: Light microscopic autoradiographic localization. Proc. Natl. Acad. Sci. USA 77: 1696-1700. 